\title{
Al-Lu'bah al-Lughawiyyah al-Namudzajiyyah fi Ta'lim al-Kitabah
}

\author{
Arif Widodo \\ Pendidikan Bahasa Arab Institut Pesantren Sunan Drajat Lamongan JawaTimur, Indonesia
}

Article History:

Received : March 6 $6^{\text {th }}, 2019$

Revised : April $8^{\text {th }}, 2019$

Accepted : May $10^{\text {th }}, 2019$

Published : June $1^{\text {st }}, 2019$

Keywords:

Games, Language, Models, Skills, Writing

* Correspondence Address: arifwidodo@insud.ac.id

\begin{abstract}
Language games of teaching Arabic and learning are important to improve the quality of learning outcomes in speaking skills. Generally, the objectives of this research are to know the importance of recreational activities by language games of Arabic. In addition, specifically, it is to know 1) writing skills, 2) language games for writing Arabic, 2) models of fun activities for writing skills. The data are collected by documentation and library research. The data are analyzed by descriptive and content analysis. The results of the research show that 1) the final directions of writing skills are to improve the ability to write official Arabic, 2) spirit, positive emotions and learning activeness can be improved with fun activities, 3) in general, there are seven models of fun activities for Arabic that can be used to improve the learning process becomes fun. The novelty of this research is the tendency of the students to be movement in the movement of comfortable activities.
\end{abstract}

$$
\begin{aligned}
& \text { اليوم، كان تعلم اللغة العربية كلغة أجنبية لناطقين بغيرها ليس من الأمر السهل. } \\
& \text { قد واجـه الدارس الإندونيسي الصعوبات العديدة حينما يتعلمون هذه اللغة التى اعتبر بها } \\
& \text { بعض الناس أصعب اللغات في العالم. وتختلف صعوبة تعلم هذه اللغة تبعا لمرحلة } \\
& \text { دراستهم وكذلك سن الدارس والبيئة التي يعيش فيها خلال تعلمه اليومي، سواء كان في }
\end{aligned}
$$


المؤسسة الرسمية أو في بيئة خارج الفصل. وكذلك تُوجد صعوبة تعلمها حسب طبيعة اللغة العربية من حيث مشابهتها أو غير مشابهها في نواحي علوم اللغة للغة الدارس الأصيلة من اللغة الإندونيسية. هناك من يسهل تعلم اللغة الإنجليزية ويصعب باللغة العربية ويشق عليه استيعابها.

وبالنسبة لتعليم مهارة الكتابة التى هي نظام من الرموز الخطية لتشكيل الأفكار

والمعارف والثقافة المتاحة لنا'، قام أيضا الدارس بالمشكلة الخاصة التى يواجهها عند دراسته حتى نحتاج إلى علاج هذه المشكلة بحلّ مناسب. والدارس في ميدان التعلم عند ما يبدأ بتعلم الكتابة فإنه يشق الكتابة الصحيحة من ناحية التراكيب والترتيبات ولايتقنها في أول المرحلة بل في نهاية المرحلة إذا يتأخر المعلم بمحاولة أن يفسر تلك المشكلات ويضع العلاج السريع. من تلك المشكلات، هناك المشكلة العامة التى يدور الدارس حولها والمشكلة الخاصة لهذه المهارة. أما المشكلة العامة فهي اختلافات خلفية لغة الدارس باللغة العربية لاسيما ثقافيته، اختلاف قدرة لغوية بين الدارس، اختلاف عاطفيته وعدد الفروق الفردية بين هؤلاء، قلة اهتمام الدارس بدراسته، قلة مشاركة الدارس في الأنشطة التعليمية في الفصل، ضعف دافعية الدارس نحو دراسة اللغة العربية وعدم توفر الوسائل التعليمية. كل منها يحدث حول تعليم وتعلم اللغة العربية بإندونيسيا.

\footnotetext{
${ }^{1}$ NajwaFauziSholeh and MarwahNashrHasan, “Atsar Al 'Al'ab Al Tarbawiyah 'AlaTanmiyahBa'dhMaharot Al Lughoh Al Arobiyah,” Al Majalah Al Jami'iyah Al Islamiyah Li Al Dirosah Al TarbawiyahWa Al Nafsiyah 1 (2018): 339.
} 
أما المشكلات الخاصة التى يواجهها الدارس حينما يتعلم في مهارة الكتابة فهي قلة التحفير يؤدي إلى استجابة الدارس من حيث كتابة الكلمة أو الجمل أو التعبيرات التحريرية العربية حسب الموضوع المدروس، طرائق تعليم الكتابة المملة، قلة إلمام المدرس بالأسـاليب الحديثة وعدم وجود الأنشطة المثيرة الترفيهية من الألعاب التى دعتٌ إلى تفعيل الدارس في عملية التعليم والتعلممع أن حقيقة الألعاب لها أهمية لتفعيل عملية التعليم إن اشتركت فيها الحواس الخمس كالسمع والبصر والشم والذوق واللمس كل أو بعض׳. ولأجل حلّ هذه المشكلة يحاول الباحث أن يعالجها بتقديم بعض نماذج اللعبة في تعليم الكتابة بالأنشطة التى تثير إلى نشاط الدارس وميله إلى روح التعلم الحقيقي لاكتساب مهارة الكتابة. وهذا يتفق بروح اللعبة أهها إحدى طرق التعليم المؤثرة على التعليم والتعلمّ؟. ومن نماذج اللعبة المقترحة هنا الحكاية المسلسلة (Chain Stories)، الكلمة العشوائية (Sig Circle) (Scrambled)، الحكمل التائّية المسلسلة (Serambled sentence)، الفقرة التأنيّة (Scrambled). كل منها لعب جيد وجديد في عالم تعلم العربية ومريح لجلب شعور عاطفي لدى الطلاب. وهذه اللعباة ستساعد على إشراك الحواس الخمس في عملية التعليم والتعلم وتخفف من رتابة الدروس وجفافهاء.

\footnotetext{
${ }^{2}$ Tarmizi Ninoersy, “AL Al'ab Al Lughowiyah Wa Tathbiiqiha Fii Ta'liim Al Muhadatsah,” Jurnal Ilmiah DIDAKTIKA X!V, no. 2 (2014): 402.

${ }^{3}$ Sri Sunarti, Ahmadi, and ZaenalIlmi, "Tatsir Al La'b Al TarbawiFiiTa'liim Al Lughoh AL Arobiyah Li Al Fashl AL Saabi' Bi AL Madrosah AL Mutawasithoh Al Hukumiyah Al NamudzajiyahBaalankarayaa," Al Ta'rib: JurnalPendidikanBahasa Arab Dan Kebahasaaraban 5, no. 1 (2017): 3-5.

${ }^{4}$ NashifMusthofa Abdul Aziz, Al 'Al'ab Al LughowiyahFiiTa'liim Al Lughoh Al Ajnabiyah, 1st ed., vol. 1 (Riyadh: Dar al Murih, 1983).
} 
ومن ناحية أخرى، هذه القضية من اللعبات المذكورة ذات خصيائص وعلاقة قوية

في ذهن الطلاب لتنمية قدرتهم على التعبيرات التحريرية بتدريب مريح يؤدي إلى الرغبة والحماسـة. وكذلك بهذه اللعبات أن تكون المادة مثيرة وممتعة ومحققة الدافعية السرية وتضمن من نشاط تعاوني مثير للعزائم لتحقيق أهداف الدرس. لذلك، هذا البحث مهم لتنفيذه لينقذ الطلبة من الملل وضيق الصيدر وما أشبـه من الإحساسات الأليمة التى تسبب الخلوة من الأعمال الجدياة.

بالنسبة لما سبق، يهدف هذا البحث إلى وصف ( ) حول تعليم مهارة الكتابة، ؟) وصف اللعبات المريحة في تعليم الكتابة، r) كيفية تعليم الكتابة عبر اللعبات المريحة. وللحصول إلى هذا البحث، قام الباحث بالبحث الأساسي لمعرفة وفهم مادة تعليم مهارة الكتابة من حيث دورها وأهمية تعليمها بطريق أكثر فعالية. ويقوم الباحث بالمدخل الكيفي في منهجية هذا البحث. بهذا المدخل حصل الباحث على البيانات من خلال الدراسة الميدانية والملاحظة خلال عملية التعليم والتعلم التى قام بها الباحث كل يوم.

الإطار النطري

\section{مفهوم مهارة الكتابة}

المهارة هي الحذق في الشيئ وفي هذا السياق يدل على صفة منهجية وعلمية ليكون

شخص متقنا والماهر هو الحاذق بكل ما فعل وعمل وأما الكتابة هي تحويل الأصوات 
اللغوية إلى الرمز وله مكان كبير في عملية التعليم والتعلمْ. والمعنى أن مهارة الكتابة هي الكفاية لفاعل على التعبير التحريري. وهي المهارة الأخيرة التى تأتي متأخرة بالنسبة لترتيها بين المهارات الأربع. وهذه المهارة تتكون من ثلاثة أنواع مختلفة وهي الرسم الهجائي والخط والتعبير الكتابي. كل من هذه الأنواع يتدرج تعليمها حسب مستويات الطلاب أو مرحلة الدراسة مثل مرحلة المدرسة الابتدائية حتى مرحلة الجامعة.

ومهارة الكتابة هذه هي عملية ذات آلي وذات عقلي. والآلي يدل على الحركية الخاصة برسم حروف اللغة العربية، معرفة التهجئة، والترقيم في اللغة العربية مثل رسم الحروف وكذلك أشكالها. أما الناحية العقلية، فيتطلب من المعارف الجيدة بكفاية النحوية، والمفردات، واستخدام اللغة استخداما فعالا وهذه هي مرحلة متأخرة عن

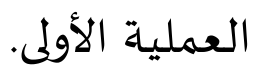
أما الكتابة اصطلاحية فهي القدرة على تصوير الفكر من الأفكار باستخدام الحروف والكلمات والجمل وفقرات حسب رأي النحو، الأسلوب الرسمي والشكل المتناسق والمظهر الجميلة. كل منها لأجل تعميق التفكير. ومن ناحية أخري هناك مستويون قام بتعريف هذه المهارة. فالأول من المستوي الظاهر وهو يشير إلى أن الكتابة التى يهتم بها الإنسان هي الخط والتهجي وتنظيم الجمل والفقرات والترقيم أو التهميش. والثاني من المستوي الباطن

${ }^{5}$ BadruzZaman, “Ta'liimMaharoh Al Kitabah Li GhoiriNathoqiina Bi AL 'Arobiyah,” Jurnalllmiah DIDAKTIKA XIV, no. 1 (2013): 128-42. 
وهو يشير إلى أن الكتابة التي يهتم بها الناس هي المحتوي وبنية الأفكار وذلك من عملية

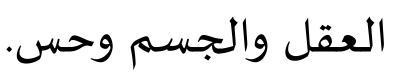

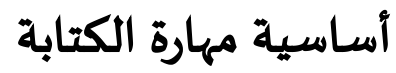

الكتابة مهمة في تطبيق اللغة العربية تحريريا. بدون المهارة في الكتابة لايمكن أن

يكتب ما المسموع أو ما المقروء كتابة صحيحة يفهمها الإنسان حينما يقرأ ذلك المكتوب. الكتابة أمر ضروري وهي وسيلة من وسائل التواصل التى يمكن بواسطتها أن يعبر الطالب عن أفكاره ومشاعره وما في باله. ويرى شحاتة أن الكتابة الصحيحة عملية مهمة في التعليم والتعلم على اعتبار أنها شيئ أساسي من عناصر الثقافة وضرورة اجتماعية لنقل الأفكاروالتعبير عن تلك الأفكاروالإلمام بهاّا. وبالنسبة لهذه الكتابة، هناك قدرة الكتاب الثلاث وهي تتركز على ثلاثة أمور وهي القدرة على الإملاء اي الكتابة الجيدة إملائيا، والقدرة على الخط اي جودة الكتابة خطا، والقدرة على التعبير التحريري عن الأفكار والمشاعر. بدون تلك القدرة، تكون الكتابة من رموزها مضطربة ومستحيلة القراءة اي صعوبة فهم المكتوب للقراء. إضافة إلى ذلك، هناك مشكلات الكتابة العربية العامة التى يواجهها الطلاب. ومن أبرز هذه المشكلات هي الشكل، وقواعد الإملاء، واختلاف صورة الحرف باختلاف موضعها

${ }^{6}$ Hasan Syahatah, Ta'lim Al Lughoh Al Arobiyah Baina Al Nazoriyah Wa Al Tathbiiq (AL Qohiro: Al Dar al Mish al Lubnaniyah, 2000). 
من الكلمة، والإعجام، ووصل الحروف وفصلها، والإعراب. تلك المشكلات يمكن حلها بتعويد التدرببات الكتابية من الإملاء والخط والتعبير حتى نجد صحتها بهذه التدريبات.

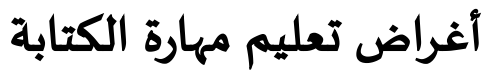

عبر هذه المهارة، يهدف منها أن يتمكن الطلاب من الاستطاعة أن يقدر على :

كتابة الحروف والكلمات والجمل والفقرات بخط وفقا لقواعد الإملاء وعلامات

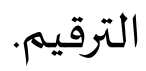

ب) تكميل الجمل العربية والفقرات العربية بعناية الكلمات المناسبة. ج) كتابة الجمل بأساليب صحيحة بصغة فصيح د) يتابة الفقرات العربية بعناية الجمل المفيدة . ( ) كتابة الأفكار من الأخبار أو المعلومات. و) كتابة الإنشاء بعناية معرفة الأفكار المضمونة في العقل ي) كتابة التحليلات والنشرات والاعلانات والتقارير والخطب والقصص الكتابية.

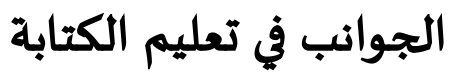

\section{اللعبة بالأنشطة التعليمية في مهارة الكتابة}

عرف كثير من المعلين والباحثين اللعب، منهم الحيلة التي عرفت بأنه نشاط حر يتوجه إلى تنمية سلوك المتعلمين من الأطفال وشخصيتهم، ويشير لحسن الصغار وتربيتهم 
من قبل كبارهم، وهو يؤدي إلى المتعدة والتسلية كما في الألعاب الشعبية والمحوسبة.` وكذلك عرفته النخالة بأن اللعب هو نشاط موجّه أو غيره يكون على نمط حركة أو نمل عمل بعملية فردية أو فريقية.^من ذلك التعريف عرف الباحث أن اللعب نشط لتحقيق أهداف ما من المادة التعليمية لأجل تطوير لغة الطلبة في عملية التعليم والتعلم. وبالنسبة لتعليم اللغة، كان اللعب اللغوي وسيلا جديدا استفادته برامج تعليم اللغات. وهذا كما بينه اللبابيدي. وكذلك إن اللعبة اللغوية وسيلة لتنمية مهارات الطلاب لأها توفر الممارسات خاصية وسيلة فعالة لترقية مهارة اللغة الشفهية والتحريرية.' 'وزاد عمر وبحر الدين من ذلك نتيجة من بحثهما أن تطبيق اللعب اللغوي يؤثر في ترقية مهارة الطلاب في الصف الثامن بالمدرسة المتوسطة بدماك.'"وبين أيضيا أن الألعاب اللغوية هي صممت لمساعدة الأطفال على اكتساب مهارات اللغوية عن طريق

\footnotetext{
${ }^{7}$ Mar'a al Hailah, Al Manahij Al Tarbawiyah Al Hadits (Oman: Dar al Masiroh, 2005).

${ }^{8}$ Samiyah al Nakholah, Al Mu'allim Wa Al Ta'allum Bi Al'ab (Gaza: Maktabah al Kulliyah al Jami'iyyah, 2008).

${ }^{9}$ Qosim al Bari, “Atsar Istikhdaam Al Al'ab Al Lughowiyah Fii Minhaaj Al Lughoh Al Arpbiyyah Fii Tanmiyah Al Anmath Al Lughowiyah Laday Tholabah Al Marhalah Al Asasiyah," Al Majalah Al Urduniyah Fii Al Ulum Al Tarbiyah 7, no. 1 (2011): 24.

${ }^{10}$ Hasyim 'Asy'ari, “Al Al'ab Al Lughowiyah Fii Ta'liim Al Qiro'ah,” Al Qodiri:Jurnal Pendidikan, Sosial Dan Keagamaan 4, no. 1 (2013): 1.

${ }^{11}$ Umar and Bahruddin, "Tathbiiq Al La'b Al Lughowi Bithoqoh Al Shodri Fii Ta'liim Al Lughoh Al Arobiyah Li Tarqiyah Al Maharoh Al Kitabah,” Arabiya: Jurnal Pendidikan Islam 10, no. 1 (2018): 122.
} 


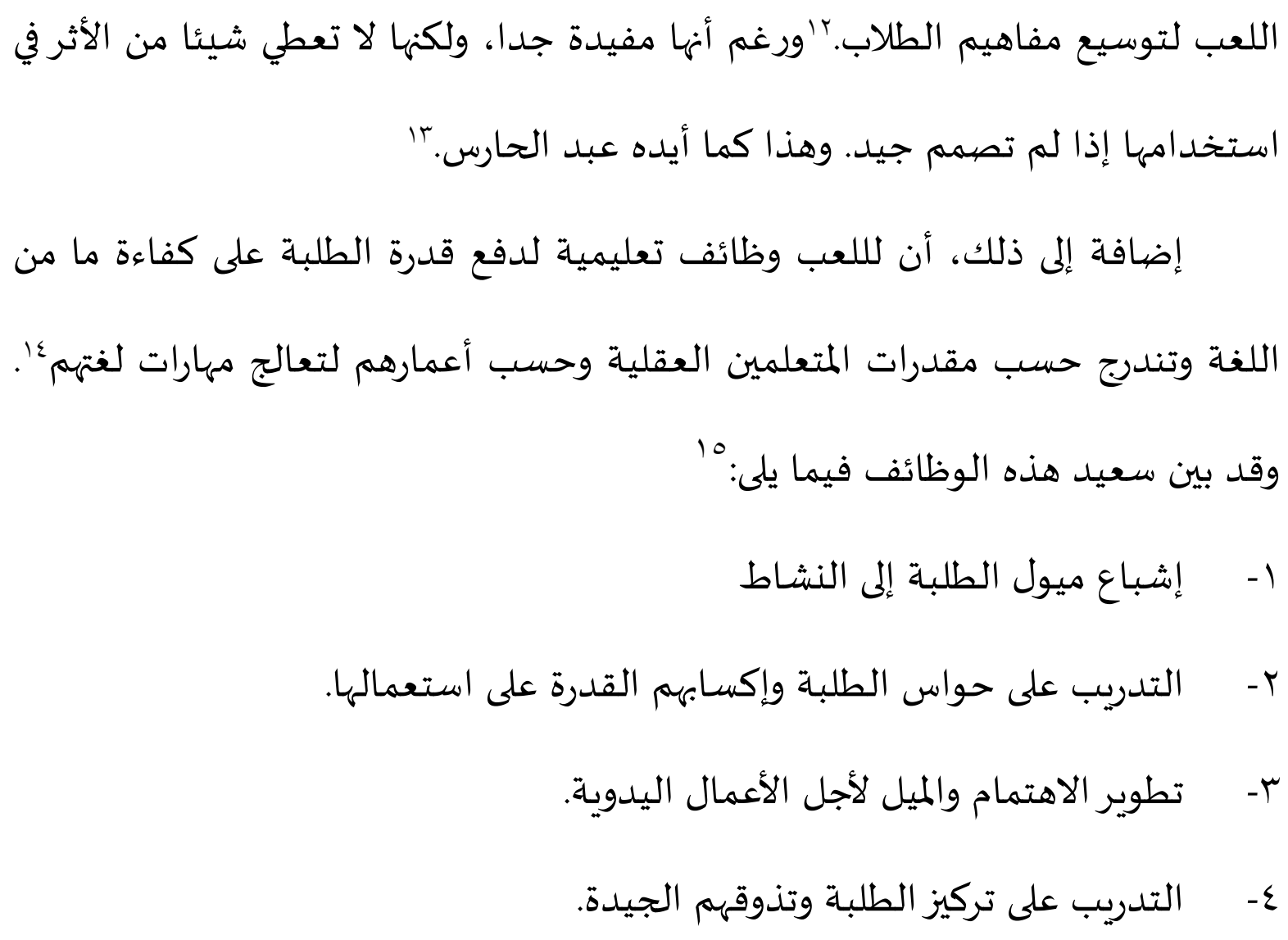

أماالنشاط التعليمي فهو مجموعة من الأفعال التى سيتم تنفيذها من خلال عملية التعليم والتعلم للوصول إلى الأهداف الموضيوعة في مرحلة التعليم الأساسي. وبين

\footnotetext{
${ }^{12}$ Nur Syamah, "Al 'Al'ab Al Lughowiyah Fii Ta'liim Al Lughoh Al 'Arobiyah (Al Nazoriyah Al 'Amah Li Istikhdam Al 'Al'ab Fii Ta'liim)," Nabighoh: Jurnal Pendidikan Dan Pembelajaran Bahasa Arab 16, no. 2 (2017): 257.

${ }^{13}$ Abdul Haris, “Al Ta'liim Al Mabni 'Ala Al Lu'bah Al Lughowiyah (Tiknulujiya Al Ta'liim Al Muqtaroh Li Ta'liim Maharoh Al Kalam Fii Al Mustawa Al Tsanawy)," Lisaniyat 1, no. 1 (2010): 3.

${ }^{14}$ Abdul Rahman, "AL Al'ab Al Lughowiyah Fii Ta'liim Al Lughoh Al Arobiyah Fii AL Madaris Al Ibtidaiyah Fii Barnaamaj Jei Qof Fii Maliziya: Dirosah Tahliiliyah," Arabiyat: Jurnal Bahasa Arab Dan Kebahasaan 2, no. 2 (2015): 279.

15, AthifSa'id and Muhammad Abdullah, الدراسات الاجتماعية: طرق التدربيس والاستر/تيجيات (AL Qohiro: Dar al Fikr al 'Arobi, 2008).
} 
السعدي أن الأنشطة التعليمية ضمن مناهج تعليم المواد الدراسية بصورة أكثر فاعلية تصل إلى جودة العملية التعليمية.17

ومن الضروري كمعلم أن يخطط بعض الأنشطة الممتعة للدارس لاكتساب لغته

الأجنبية عن طريق سهلة وقلة مملة. وبالأنشطة المناسبة التي سهل تنفيذها وتطور ابتكار

الدارس ومواهبه لتدعيم ميوله ورغبته في التعلم وجعل عملية التعليم والتعلم ممتعةً ومشوقة لدياه حتى تؤدي إلى زيادة المهارات التعليمية فضلا عن مهارة الكتابة.

وبالنسبة لهذه المهارة، هناك بعض اللعبات المريحة المناسبة بتعليم الكتابة. وتلك

اللعبات المريحة سيراها الباحث من حيث علم اللغة وعلم النفس الذي يُؤكِد ويشّدّ هذه

اللعبة. وذكرت رحمة خمس أنشطة من اللعبات المريحة تناسب في تعليم مهارة الكتابة،

وهي () نموذج التعليم والتعلم عبر الحكاية المسلسلة (Chain Stories)، ؟) نموذج التعليم

والتعلم عبر الكلمة العشوائية (Scrambled)، ب) نموذج التعليم والتعلم عبر الحكاية

المسلسلة (Big Circle)، ع) نموذج التعليم والتعلم عبر الحكاية المسلسلة ( Serambled

IV.Chain Stories (sentence)، نموذج التعليم والتعلم عبر الحكاية المسلسلة.

بالنظر إلى نماذج اللعبة المذكورة يرها الباحث أن هذه اللعبة تثير عمليتها في

عاطفية الطلاب في المدارس بل الجامعات. وبهذه اللعبات ترتفع كفائتهم وقدرتهم اللغوية.

\footnotetext{
${ }^{16}$ Fatimah Binti Mahmud al Sa'dy, "Fa'aliyah Wihdah Al Qoimah'Ala Nasyaath Fii Tafshili Tholabah Al Shoff Al Tsalis Li 'Idad Fii Madah Al Tarikh" (Al Bahs al Masjitiri, Jami'ah Sulthon Qobus Malik Oman, 2001).

${ }^{17}$ ZuliatiRahmah, Fun Activities (Malang: Bintang Sejahtera, 2013).
} 
ذلك بالنسبة لمذهب علم النفس أن العوامل الخارجية تؤثر في ترقية كفاءة تعلم اللغة.^وكذلك العوامل الداخلية تؤثر في تحمُّس الدارس في عملية التعليم والتعلم. والمذهب الذي اعتمد على هذه العوامل هو المذهب المعرفي (عصيلي، دون سنة).

\section{منهج البحث}

منهج البحث هو طريقة تحليل مشكلات البحث التى تجري مترتبة لحصول على حقيقة المسألة والنتائج كي يفهم ويتبحر الباحث عن حال بيئة البحث. والمنهج في هذا البحث مقصود باه لتعميق ومعرفة موضوع البحث المرجوّ.جإان المنهج الذي يستخدماء البحث في هذا البحث هو المنهج المناسب الذي هو كيفية عامة لجمع المعلومات وتحليلها وهو يتركز على البحث الوصفي. وعملية هذا البحث ينقسم إلى ثلاثة مراحل: نشاط قبل ميدان، نشاط في ميدان بالعمل التطبيقة، وتحليل البيانات. وأما نوع هذا البحث فهو البحث الوصفي. وبنوع هذا البحث، يحاول الباحث أن يحل المشكلة المتعلقة بتعليم مهارة الكتابة بطريقة وصف اللعبات المستخدمة في تعليم مهارات اللغة الأبع. ولتحقيق هذا العمل، سيأخذ الباحث البيانات الطبيعية من قبل باشتراكه مباشرة في ميدان البحث لحصول على تلك البيانات. ‘وهذا البحث كما بين على

\footnotetext{
${ }^{18}$ Ahmad FuadEffendy, MetodologiPengajaranBahasa Arab (Malang: Misykat, 2005).

${ }^{19}$ AR.Syamsudin and S. Vismaya, MetodePenelitianBahasa (Bandung: RosdaKarya, 2007).

${ }^{20}$ SuharsiniArikunto, ProsedurPenelitianSuatuPendekatanPraktek (Jakarta: RinekaCipta, 2002).
} 
إدريسلحصول على المعلومات بدون استغال بالتطبيق الفعلي وذكر أن هذا البحث من

البحث الأساسي ضد البحث التطبيقي. لذلك استخدم الباحث في منهجهاه بحثا مكتبيا."r

نتائج البحث

اطلاعا على ما تحدث الباحث حول الكتابة وتعليمها، وجد الباحث أن في تعليم

الكتابة ما يجب أن يلتفت إليه المعلم وهو اللعبة اللغوية. اللعبة اللغوية هي وسيلة

لتوصيل الطلاب النجاح كما أوصي صبري ببحثه في اللألعاب اللغوية المحوسبة أن المعلم

يتطلب مناه تصميم الألعاب اللغوية لتنمية مهارات اللغة العببية.r

كثير من نتائج البحث يدل على فعالية اللعبة في إنماء كفاءة الطلبة على مهارات

اللغة العربية. واللعبة في هذا البحث جزء من لعبات قام بها المعلم في تنشيط عملية

التعليم والتعلم في تعليم اللغة العربية، وهي ذات خصائص وكيفية التقديم كي تستفيد

منها في جلب الأثر. وقد عرف أن اللعب يشير إلى عمل ممتع ونشاط مثمر وإعداد فعال للحياة المستقبلة. ّ كل من ذلك حسب لعبة صممها المعلم وتنويع نماذجها بالنسب إلى مرحلة الطلبة وخصائصهم.

${ }^{21}$ Ali Idris, Madkhol Ila Manahij Al Bahs Al 'Ilm Li Kitabah Al Rosaiail Al Jami'iyyah (Al Qohiro: Al Dar al Arobiyyah Li al Kitab, 1985).

${ }^{22}$ Sobri Bin Syahrir, "'Al 'Ab Al Lughowiyah Al Mahusabah Fii Ta'liim Al Lughoh Al Arabiyah Li Ghoir Al Nathiqina Bi Ghoiriha Baina Al Nazoriyah Wa Al Tathbiiq," Journal of Faculty of Dar Al Uloom, 2013, 12.

${ }^{23}$ Ahmad Ibrohim Shouman, “Atsar Istirootijiyah Al 'Al'ab Al Lughowiyah Fii Tanmiyah Maharoot Al

Takhoyyul Al Ibda’iy Laday Athfaal Al Roudhoh Fii Madinah Omman” 11, no. 2 (2016): 157. 
إضافة إلى ما ذكر الباحث، أن نجاح عملية الألعاب في الدرس اعتمد على نموذج

التعليم والتعلم المتنوع والعديد حتي يحرك ميول الطلبة وإرادتهم. ذلك لإن الألعال كما بين نور عيني ستعالج كل المهارات الأساسية من مهارات اللغة الأربعء وبالقول الآخر في حاجة إلى استراتيجية مناسبة بهذه اللعبة المستخدمة. وبهذا الصدد تعرّف استراتيجيّة التعليم بأنّها مجموعة من الإجراءات من قِبل المعلم لينفذها في عمليّة التعليم بطريقة مناسبة. والهدف من استخدام الاستراتيجيّة هو لرفع جودة عمليّة التعليم إلى أعلى مستوى يرجو باء المعلم.

بالنسبة إلى الأنشطة الترفيهية تقدم في هذا البحث استراتيجية رائعة للإستمتاع بمجموعة متنوعة من الأنشطة التفاعلية المريحة التي تحسن مهارات الكتابة لدى الدارس أو من يريد تعلّم اللغة العربية. هذه الأنشطة مثالية جداً لكثير من دارسي اللغة العربية التي يودُون أن يتمتعوا بالكتابة وكذلك للمعلمين الذين يبحثون عن مصادر ممتعة لتعليم مهارة الكتابة الفعالية والتفاعلية. وفقا لما سبق، فيما يلى شرح نماذج اللعبات في تعليم الكتابة :

نموذج التعليم والتعلم عبر الحكاية المسلسلة (Chain Stories)، قبل بداية اللعبة، جهّز الطالب القرطاس والقلم. وأما الأنشطة بلعبة الحكاية المسلسلة تتكون من ست خطوات، وهي الأول أن يجلس الطالب مدورا يحمل الورق من القرطاس وكذلك القلم. كل من

${ }^{24}$ NurAini, "Istirootijiyah Al Al'ab Al LughowiyahFiiTa'liim AL Lughoh Al Arobiyah," JurnalAnNabighoh 17, no. 1 (2015): 74-77. 
الطلاب يكتب اسمه الأصلي على المكتب. والثاني ينبني للمعلم أن يعطي الإشارة في بداية الكتابة بإشارة خاصة مثل رفع يديه أو بتصفيق اليد. في البداية، يشير المعلم بالإشارة (تصفيق اليد) لكي يكتب الطالب شيئا من الجملة العربية تحت كتابة الاسم. ولكل واحد من الطلاب الفرصة المحدد الذي عينه المعلم حتى لو أشار المعلم إشارة ثانية، دلّ أن وقت الكتابة قد انتهي. والثالث أن يسلم أو يعطي الكاتب من الطلاب كتابته المكتوبة من الجمل صديقة بجانبه الأيسر. وبعد كتابته الأولى وبعد إعطاء الورق لصاحبه، أشار المعلم إشارة تدل على بدء الكتابة الثانية. وكتب الطالب كتابة ثانية بعد الإشارة من المعلم وكتب شيئا من الجملة المختلفة بالكتابة الأولى بجانب كتابة صاديقه الأيمن الذي يعطيه الورق فيه كتابته أولى. وبعد انتهاء هذه الكتابة، يعطى الطالب الورق صديقة مرة ثانية. ويجري هذا الحال حتى يرجع ورق الطالب الأول إلى صاحبه الأصيل. والرابع، يقرأ

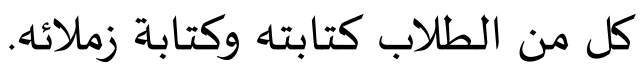

بعد إجراءات ما ذكر، أمر المعلم أحد الطلاب أن يتقدم ويقرأ ما المكتوب في القرطاس. حينئذ، أتاح المعلم الفرصة كلا منهم لتقديم الآراء والاقتراحات وكذلك تعليقات تؤدي إلى تصليح كتابة ما من ناحية القواعد والإملاء العربي. وفي الهاية، صحح المعلم الأخطاء وبين علوم ذلك الأمر. نموذج التعليم والتعلم عبر الكلمة العشوائية (Scrambled)، أول ما فعل الطالب في هذه اللعبة أن يجهّز قطعة القرطاس، القلم، الجريدة أو المجلات. وأن يستعدّ بقطعة 
الكلمة المأخوذة من الكتاب أكلمة أو جريدة أو مجلات فهها كتابة عربية عن شيئ ما. ويُدخل الكاتب في الفلاف قطعة الكلمات التى يمكن بها تكوين الجملة المفيدة. يجعل الطالب الرقم ويكتبه على الغلاف واكتب الجملة المفيدة كالجملة المفتاحية وفق الأرقام المعينة. وأما الخطوات في هذه اللعبة فهي : الأول أن يضرب المعلم مثلا عن كيفية ترتيب الكلمة. فليعطهه المعلم بعض الكلمات المأخوذة من أحد الغلافات. وبعد ذلك، اكتب تلك الكلمات على السبورة واطلب من الطلاب أن يرتها بكتابتها على السبورة. والثاني، بعدما تنتهي الكتابة، فتش تركيب الجملة من حيث كتابة الصحيحة أو الخطيئة لأن ذلك إما أن يكتب صحيحا وإما أن يكتب خطأ. إضافة إلى ذلك، من الأحسن أن يأتي المعلم الهدية لمن وصل إلى النجاح في تركيب الجمل المفيدة. وذلك لجلب ميولهم وحماشتهم في عملية التعليم والتعلم. والثالث، بعد أن يعرف ويفهم الطلبة عن كيفية ترتيب الكلمات بتركيب الجملة المفيدة، قسم المعلم أربع مجموعات مختلفة بحمل الغلاف وضع فيه المعلم كلمات جديدة. هذه المجموعات تستبق في تركيب الجمل الصحيحة. والمجموعة التى تمّ عملهم كتبها في الورق ويسلم وظيفتهم إلى المعلم لأجل التصحيح. حينئذ، لو صحّ التركيب، زاد المعلم بعض كلمات أخري لترتيها مرة ثانية، ولو أخطأ في الكتابة، عدّ مرة ثانية لكي يصحّ تركيبها. 
في هذه الخطوة، يسابق الطلاب من المجموعات سرعة التركيب وترتيب الكلمات إ لى الجمل المفيدة. وفي هذا الصدد، يمكن المعلم أن يزيد درجة التدريبات بترتيب الجمل إلى الفقرات الجديدة حسب الجمل المقطعة.

نموذج التعليم والتعلم عبر الحكاية المسلسلة (Big Circle)، في هذه اللعبة، أعدّ الطالب قطعة من الصور، القرطاس والقلم. أعد الطالب قطعة الصهورة بالنسبة لنوع الجمل التى يوده المعلم من الطلاب. لو تريد أن يكتب الطالب تعريف الأشياء من المباني، قام بإعداد صور البناء المختلفة مثل صورة الجامعة، صورة الشركة، صورة المدارس، صورة الشقة وهلم جرى. ولو تريد أن يكتب الطالب صورة الأنشطة اليومية، قام بإعداد صور الرياضية، التعلم، السباحة، الصيد وغير ذلك.

وأما الخطوات من هذه اللعبة فهي أن يأمر المعلم أن يُعدّ الطلاب جالسين مدورا بحمل القرطاس والقلم. وأن يشرح المعلم أن الطلاب سيكتبون جملة واحدة لكل صورة مسكوها. ولاينسي أن يضرب لهم مثلا في جعل الجملة حسب صورة ما. والخطوة الثاني أن يوزع المعلم صورة لكل واحد من الطلاب. اطلب منهم أن يكتب جملة بعد تصفيق اليد واطلب بتوقف بعد تصفيق اليد الثاني.

وبعد ذلك، أن يعطي كل واحد صورته لزميله الأيمن ثم كتب مرة ثانية جملة

حسب صهورة ما. ذلك يجري مثل ما جري حتى يجد كل واحد صورته الأولى. ومن الممكن أن يأمر المعلم طلابه بكتابة الجملة من صورة ما بل يكتب الفقرة الاساسية. 
نموذج التعليم والتعلم عبر الجمل التأّنية (Serambled sentence)،ورالأدوات المحتاجة في هذه اللعبة هي قطعة من كلمات عديدة. وهذا المصطلهج مشهور أيضا باسم الكلمة المتقاطعة من إحدى لعبة فكرية تعتمد على جمع حروف لتكوين كلمات ب. وكانت الكلمات المتقاطعة اشهر الألعال التى يمارسها الناس واكثر الاتشار في العالم وهي من لعبة فكرية وذهنية. وينقسم الفصل العربي إلى ست مجموعات ويوزع لكل مجموعة قطعة تلك الكلمات التى وضع في الغلاف. والخطوات التى سيقوم بها المعلم والطلاب فهي مالي : ا ـ كل من الطلاب يرتب كلمات في الغلاف. ويهتم الطلاب بالمعلومات الخاصة دلّت

$$
\text { على موضعها هل هي في أول الجملة أم آخرها. }
$$

Y. لكل من الطلاب أن يتجول حول زملأهم لنظر عمل زملاههم في ترتيب الجمل. ويعطي المعلم أن يكرر في كتابته قبل جمعه إلى المعلم. ". واطلب من الطلاب أن يقرأ الفقرة التى كتبها في الغلاف ويعطيها إلى الأمام. ؛ ـ ويمكن أن يكرر الخطوات لترتيب الجمل الجديدة في جعل الفقرة الجديدة. وأما الجمل المرتبة إما من القصة القصيرة، الحوار، الشعر، قعطة من قصص في الجريدة أو المجلات. وذلك الفقة من ناحية الموضوع مفوض لكل طالب أو يعين المعلم.

\footnotetext{
${ }^{25}$ Bukhori Muslim and RaudhahMarzuki, "IstikhdamWasilah Al Kalimaat Al Mutaqothi'ahFiiTadriis Al Imla AL Istima'i Li TarqiyahQudroh Al Tholabah 'AlaTarkiib Al Jumal AL Mufiidah Bi Ma'hadDaar Al Ihsan," JurnalLisanuna 7, no. 1 (2017): 3.
} 
نموذج التعليم والتعلم عبر الفقرة التأنّية (Scrambled)،في هذه اللعبة قسم المعلم

الفصل إلى خمس مجموعات. ويتركز هذا العمل إلى ترتيب الفقرة لمراجعة كفايتهم أو لتعويد كتابة الجملة العربية إلى الفقرة العربية من تلك الجمل. والطريقة الأسـاسية من هذا النموذج هي أن يأمر المعلم طلابه بترتيب قطعة الفقرات التى وزعها المعلم.

وبعد ما قام بهذا العمل، من الأحسن أن يتقدم أحد من الطلاب لتفتيش أو نظر

عمل زميله في جعل الفقرة من الجمل المقطعة. وإضافة إلى ذلك أن يضع المعلم قطعة من الجمل في زوية الفصل ويقوم كل من الطلاب بأخذ تلك القطعة. بالنسبة لنماذج الألعاب المذكورة، رأي الباحث أن كل لعب مع تنوّعه الذي قد سبق ذكره يساعد كثيرا من الدارسين. وهذا يدل على أن الألعاب اللغوية كما شرح إنامي تعين على مواصلة الجهود ومساندتها والتخفيف من الرتابة وجفاف عملية التعليم والتعلمج"r ومن ناحية أخرى، اللعب هنايتجها إلى تنشيط الطلبة عقليا ونفسيا وعاطفيا وميلا اي دافعيا. والدافعية هذه تنشأ بسبب العوامل المتولدة من اللعبة المتنوعة لأها مجموعاة الظروف الداخلية والخارجية المتحركة من أجل التوازن وحركتها الخارجية بسبب العوامل الخارجية التى يشعر الطلبة بمتعة في تعلمها.rv

\footnotetext{
${ }^{26}$ MohInami and PutriZunitaShara, "Wasilah Al Lu'bah Al Lughowiyah Broken Square Wa Snow Balling FiiTa'lim Al Lughoh Al ‘Arabiyyah Li TarqiyahMaharoh Al Qiroah,” Jurnal Al Bayan 10, no. 2 (2018): 23033.

${ }^{27}$ Abdul Nashr al Jaroh, “Atsar Al Tadriis Bi Istikhdaam Barmajiyah Ta'liimiyah Fii Tahsiin Daafiiyah Ta'allum Al Riyadhiyat Laday Tholabah Al Shof Al Tsani Al Asasi Fii Al Urdun” 10, no. 3 (2014): 274.
} 


\section{الخلاصة}

نظرا لما سبق في تنشيط عملية التعليم والتعلم باستخدام اللعبة المريحة هذه،

رأي الباحث أن هذا البحث يشير إلى الجميع أن دور الأنشطة المريحة تثير إلى فعالية الدرس وترقية ميول الطلبة. واستنتج الباحث من هذا البحث بأن تعليم مهارة الكتابة سيكون ذا أثر لدي الدارسين اعتمادا على كيفية تعليم هذه المهارة الكتابية، ومملة التعلم كانت تنبت بسبب قلة اللعبة المريحة الداعية إلى تفعيل الطلاب في عملية التعليم والتعلم. وكانت العاطفة السلبية يمكن علاجها باللعبة المريحة لجذب ميول الطلاب في تعلم مهارة الكتابة. ونماذج اللعبة المريحة من حيث استراتيجية تنفيذها يمكن أن يطور الطالب بتطويرها حسب احتياجات التعلم في عملية التعليم والتعلم في الفصل. وبالنسبة لذلك، أوصي الباحث لكل معلمي اللغة العربية أن يتمسك بالأنشطة الدافعة إلى تفعيل عملية التعليم والتعلم حسب مادة سيعلمها في الفصل. ذلك لأن ميول الطلبة ورغبتهم في الدرس قد تتأثر بوجود العوامل الخارجية مثل اللعبات الفعيلة.

Abdul Aziz, Nashif Musthofa. Al 'Al'ab Al Lughowiyah Fii Ta'liim Al Lughoh Al Ajnabiyah. 1st ed. Vol. 1. Riyadh: Dar al Murih, 1983.

Aini, Nur. "Istirootijiyah Al Al'ab Al Lughowiyah Fii Ta'liim AL Lughoh Al Arobiyah." Jurnal An Nabighoh 17, no. 1 (2015): 74-77. 
Arikunto, Suharsini. Prosedur Penelitian Suatu Pendekatan Praktek. Jakarta: Rineka Cipta, 2002.

AR.Syamsudin, and S. Vismaya. Metode Penelitian Bahasa. Bandung: Rosda Karya, 2007.

'Asy’ari, Hasyim. “Al Al'ab Al Lughowiyah Fii Ta'liim Al Qiro'ah.” Al Qodiri:Jurnal Pendidikan, Sosial Dan Keagamaan 4, no. 1 (2013): 1.

Bari, Qosim al. “Atsar Istikhdaam Al Al’ab Al Lughowiyah Fii Minhaaj Al Lughoh Al Arpbiyyah Fii Tanmiyah Al Anmath Al Lughowiyah Laday Tholabah Al Marhalah Al Asasiyah.” Al Majalah Al Urduniyah Fii Al Ulum Al Tarbiyah 7, no. 1 (2011): 24. Effendy, Ahmad Fuad. Metodologi Pengajaran Bahasa Arab. Malang: Misykat, 2005. Hailah, Mar'a al. Al Manahij Al Tarbawiyah Al Hadits. Oman: Dar al Masiroh, 2005. Haris, Abdul. “Al Ta'liim Al Mabni 'Ala Al Lu'bah Al Lughowiyah (Tiknulujiya Al Ta'liim Al Muqtaroh Li Ta'liim Maharoh Al Kalam Fii Al Mustawa Al Tsanawy).” Lisaniyat 1, no. 1 (2010): 3 .

Idris, Ali. Madkhol Ila Manahij Al Bahs Al 'Ilm Li Kitabah Al Rosaiail Al Jami'iyyah. Al Qohiro: Al Dar al Arobiyyah Li al Kitab, 1985.

Inami, Moh, and Putri Zunita Shara. "Wasilah Al Lu’bah Al Lughowiyah Broken Square Wa Snow Balling Fii Ta’lim Al Lughoh Al 'Arabiyyah Li Tarqiyah Maharoh Al Qiroah.” Jurnal Al Bayan 10, no. 2 (2018): 230-33.

Jaroh, Abdul Nashr al. “Atsar Al Tadriis Bi Istikhdaam Barmajiyah Ta'liimiyah Fii Tahsiin Daafiiyah Ta'allum Al Riyadhiyat Laday Tholabah Al Shof Al Tsani Al Asasi Fii Al Urdun” 10, no. 3 (2014): 274.

Muslim, Bukhori, and Raudhah Marzuki. 'Istikhdam Wasilah Al Kalimaat Al Mutaqothi'ah Fii Tadriis Al Imla AL Istima'i Li Tarqiyah Qudroh Al Tholabah 'Ala Tarkiib Al Jumal AL Mufiidah Bi Ma’had Daar Al Ihsan.” Jurnal Lisanuna 7, no. 1 (2017): 3.

Nakholah, Samiyah al. Al Mu'allim Wa Al Ta'allum Bi Al'ab. Gaza: Maktabah al Kulliyah al Jami'iyyah, 2008.

Ninoersy, Tarmizi. “AL Al'ab Al Lughowiyah Wa Tathbiiqiha Fii Ta'liim Al Muhadatsah.” Jurnal Ilmiah DIDAKTIKA X!V, no. 2 (2014): 402.

Rahmah, Zuliati. Fun Activities. Malang: Bintang Sejahtera, 2013. 
Rahman, Abdul. “AL Al'ab Al Lughowiyah Fii Ta'liim Al Lughoh Al Arobiyah Fii AL Madaris Al Ibtidaiyah Fii Barnaamaj Jei Qof Fii Maliziya: Dirosah Tahliiliyah.” Arabiyat: Jurnal Bahasa Arab Dan Kebahasaan 2, no. 2 (2015): 279.

Sa'dy, Fatimah Binti Mahmud al. "Fa'aliyah Wihdah Al Qoimah 'Ala Nasyaath Fii Tafshili Tholabah Al Shoff Al Tsalis Li ’Idad Fii Madah Al Tarikh.” Al Bahs al Masjitiri, Jami'ah Sulthon Qobus Malik Oman, 2001.

Sa'id, 'Athif, and Muhammad Abdullah. الدراسات الاجتماعية: طرق التدريب والاستر/تيجيات. AL Qohiro: Dar al Fikr al 'Arobi, 2008.

Sholeh, Najwa Fauzi, and Marwah Nashr Hasan. “Atsar Al 'Al'ab Al Tarbawiyah 'Ala Tanmiyah Ba'dh Maharot Al Lughoh Al Arobiyah.” Al Majalah Al Jami'iyah Al Islamiyah Li Al Dirosah Al Tarbawiyah Wa Al Nafsiyah 1 (2018): 339.

Shouman, Ahmad Ibrohim. “Atsar Istirootijiyah Al 'Al'ab Al Lughowiyah Fii Tanmiyah Maharoot Al Takhoyyul Al Ibda'iy Laday Athfaal Al Roudhoh Fii Madinah Omman" 11, no. 2 (2016): 157.

Sunarti, Sri, Ahmadi, and Zaenal Ilmi. “Tatsir Al La'b Al Tarbawi Fii Ta'liim Al Lughoh AL Arobiyah Li Al Fashl AL Saabi’ Bi AL Madrosah AL Mutawasithoh Al Hukumiyah Al Namudzajiyah Baalankarayaa." Al Ta'rib: Jurnal Pendidikan Bahasa Arab Dan Kebahasaaraban 5, no. 1 (2017): 3-5.

Syahatah, Hasan. Ta'lim Al Lughoh Al Arobiyah Baina Al Nazoriyah Wa Al Tathbiiq. AL Qohiro: Al Dar al Mish al Lubnaniyah, 2000.

Syahrir, Sobri Bin. “'Al 'Ab Al Lughowiyah Al Mahusabah Fii Ta'liim Al Lughoh Al Arabiyah Li Ghoir Al Nathiqina Bi Ghoiriha Baina Al Nazoriyah Wa Al Tathbiiq." Journal of Faculty of Dar Al Uloom, 2013, 12.

Syamah, Nur. “Al 'Al'ab Al Lughowiyah Fii Ta'liim Al Lughoh Al 'Arobiyah (Al Nazoriyah Al 'Amah Li Istikhdam Al 'Al'ab Fii Ta'liim)." Nabighoh: Jurnal Pendidikan Dan Pembelajaran Bahasa Arab 16, no. 2 (2017): 257.

Umar, and Bahruddin. “Tathbiiq Al La'b Al Lughowi Bithoqoh Al Shodri Fii Ta'liim Al Lughoh Al Arobiyah Li Tarqiyah Al Maharoh Al Kitabah.” Arabiya: Jurnal Pendidikan Islam 10, no. 1 (2018): 122. 
Zaman, Badruz. "Ta'liim Maharoh Al Kitabah Li Ghoiri Nathoqiina Bi AL 'Arobiyah." Jurnal Ilmiah DIDAKTIKA XIV, no. 1 (2013): 128-42. 